\title{
NEURODEGENERATION
}

\section{Tau-targeting antibody therapies: too late, wrong epitope or wrong target?}

Jabbari $\mathrm{E}^{12 *}$, Duff $\mathrm{KE}^{3} 4 *$

${ }^{1}$ Department of Clinical and Movement Neurosciences, UCL Queen Square Institute of Neurology, London, UK

${ }^{2}$ Movement Disorders Centre, UCL Queen Square Institute of Neurology, London, UK

${ }^{3}$ Department of Pathology and Cell Biology, Columbia University Irving Medical Center, New York, USA

${ }^{4}$ UK Dementia Research Institute, University College London, UK

* Corresponding authors: Dr Edwin Jabbari ${ }^{2}$ (e.jabbari@ucl.ac.uk) and Prof Karen E Duff ${ }^{3}$ (k.duff@ucl.ac.uk).

No. of words in body of manuscript (excluding title page, figure and references) $-1,154$

No. of references -12

Total number of figures -1

\section{Standfirst}

Two phase 2 studies of $\mathrm{N}$-terminal tau-targeting antibody therapy fail to show clinical efficacy in Progressive Supranuclear Palsy, despite evidence of target engagement. 
The frontotemporal dementia (FTD) spectrum of neurodegenerative diseases includes Progressive Supranuclear Palsy (PSP) which is characterised by neuronal and glial tau pathology in the basal ganglia, brainstem and cortical brain regions. The classical clinical subtype, known as Richardson syndrome, is characterised by axial and limb parkinsonism that is unresponsive to treatment with levodopa (a dopamine precursor), and progressive impairments in balance, eye movements, cognition and bulbar function ${ }^{1}$. Although no effective disease-modifying therapies for PSP have been identified to date, drug development efforts over the last decade have focused on tau as a therapeutic target. This has culminated in two separate randomized, double-blind, Phase 2 trials of monoclonal antibodies targeting the N-terminus of tau, namely Gosuranemab and Tilavonemab. The studies, published in current issues of Nature Medicine ${ }^{2}$ and Lancet Neurology ${ }^{3}$, both failed to show clinical efficacy despite evidence of target engagement.

Tau is encoded by the microtubule associated protein tau (MAPT) gene, and mutations at numerous locations within MAPT are linked with familial FTD. Tau pathology is also one of the pathognomonic features of Alzheimer's disease (AD). In cerebrospinal fluid (CSF) from AD patients, tau is detected as fragments containing mostly $\mathrm{N}$-terminal epitopes ${ }^{4}$. Extracellular, unbound $\mathrm{N}$-terminal tau was proposed to mediate cell-to-cell transmission of toxic tau species, providing a rationale for the use of gosuranemab and tilavonemab ${ }^{4}$; it was hypothesised that removing unbound $\mathrm{N}$-terminal tau from the extracellular space via passive immunotherapy would slow the progression of disease. Preclinical testing of the antibodies in vitro and in tau transgenic mouse lines has been limited, but has provided some evidence that engagement of Gosuranemab reduced unbound N-terminal tau in CSF and interstitial fluid ${ }^{5}$, while Tilavonemab administration was shown to attenuate both tau pathology and neurodegeneration in vivo ${ }^{6}$. Additionally, a 12-week phase 1 multiple ascending dose study of Gosuranemab in patients with PSP showed a favourable safety profile and clear evidence of target engagement as shown by a reduction in CSF unbound $\mathrm{N}$-terminal tau by an average of $90 \%$ at all doses $^{2}$, offering hope of clinical efficacy in the subsequent phase 2 studies.

The phase 2 studies of Gosuranemab ( $N=486)$ and Tilavonemab ( $N=377)$ were well-designed trials that were adequately powered to detect a clinically meaningful difference in the total PSP rating scale score at 52 weeks, with treatment versus placebo (the primary outcome measure). In both trials, treatment and placebo groups were well matched in terms of demographics, clinical characteristics and safety profile. The Gosuranemab trial was completed but failed to show efficacy, while the Tilavonemab trial was terminated early after pre-specified futility criteria were met at the second interim analysis. There was also a lack of efficacy across a number of secondary endpoints 
including clinical, imaging and fluid biomarker outcome measures. Although the two trials used differing clinical diagnostic criteria for PSP, both studies required patients to fulfil criteria for the classical Richardson syndrome subtype. Of note, in both trials, the 1-year change in total PSP rating scale score was around +11 points, consistent with previous natural history studies of PSPRichardson syndrome ${ }^{1}$. Importantly, pharmacokinetic data for both trials suggest that the drug reached the CNS, while target engagement was again shown by a reduction in CSF levels of free, unbound N-terminal tau. In the Tilavonemab trial, a corresponding significant increase in total plasma tau was detected, which the authors hypothesise may be due to a treatment-induced increase in the peripheral tau pool. A recent publication of neuropathological findings in three patients from the Gosuranemab trial revealed that there was no evidence of tau pathology clearance. Gosuranemab administration may be associated with an unusual glial response which included accumulation of tau within perivascular astrocytic lysosomes ${ }^{7}$, but the significance of this observation is unknown.

Despite evidence of target engagement, both trials failed to show clinical efficacy and there are a number of potential reasons for this outcome. Firstly, the distinct lack of an objective non-clinical diagnostic biomarker of early PSP pathology and the significant clinical overlap with other neurodegenerative diseases such as Parkinson's disease means that a clinical diagnosis of PSP is often delayed. Indeed, in both trials, the disease duration at the time of enrolment was around 3 years while the PSP rating scale score was 36 , corresponding to a moderate stage of disease ${ }^{1}$. By this stage, it may be too late for tau reduction to be effective as a disease-modifying strategy. Although an objective early diagnostic biomarker of PSP remains elusive, the inclusion of slower progressing non-classical PSP clinical subtypes with a milder density of tau pathology ${ }^{8}$ may lead to a more favourable outcome in future tau-targeting trials. However, Gosuranemab was clinically futile at 78 weeks in a large $(n=654)$ phase 2 trial of mild AD patients, supported by CSF and PET biomarkers of early $A D$ pathology ${ }^{9}$. It is therefore entirely possible that even early stage disease is too late, and that the pre-clinical phase is the optimum time to intervene.

Secondly, the N-terminal region of tau is now considered unlikely to be the mediator of tau toxicity ${ }^{4}$, and the paucity of preclinical data supporting this target may render its failure in human trials less surprising. More recent trials are investigating agents that target epitopes in the mid-domain of tau (Figure 1), including the microtubule-binding region, which are abundant in human CSF and, along with C-terminal tau fragments, are considered to be more pathological than $\mathrm{N}$-terminal fragments ${ }^{4}$. While there has been intense focus on the pathogenic role of soluble tau oligomers, it remains 
unclear which specific tau species are toxic ${ }^{4}$. There are a number of ongoing disease-modifying trials in $A D$ focusing on passive immunotherapy using tau antibodies against a range of epitopes (Figure 1), alongside active immunotherapy trials. Alternative tau-lowering approaches that are agnostic to the form of pathogenic tau include antisense oligonucleotide therapy which is currently in phase 1 trials in $A D^{4}$.

Thirdly, we must also be open to the possibility that tau, especially extracellular free tau, may be a less effective therapeutic target than upstream dysfunctional biological pathways that are driving disease progression and the ongoing accumulation of tau pathology. Recently, genetic and functional studies have implicated the role of the LRRK2 gene in survival of patients with $\mathrm{PSP}^{10}$ and tau uptake in human neurons ${ }^{11}$. Additionally, proteostasis (especially the ubiquitin proteasome system) has been implicated in determining PSP clinical phenotype ${ }^{8}$ and as a modulator of pathogenic tau accumulation in vivo ${ }^{12}$. It is possible that drugs acting on these pathways, either alone or in combination with tau-targeting therapies, will be needed to effectively modify the progression of tau-related diseases. The design of these future trials may also involve genetic stratification to identify patients who would potentially benefit most from these approaches.

Finally, the study of large, deeply phenotyped longitudinal cohorts with long-term outcomes, including survival, is central to the further discovery of novel biological determinants of disease progression and therapeutic targets. The Gosuranemab and Tilavonemab trial cohorts are rich datasets which provide such opportunities-realizing their potential should be a priority for the scientific community.
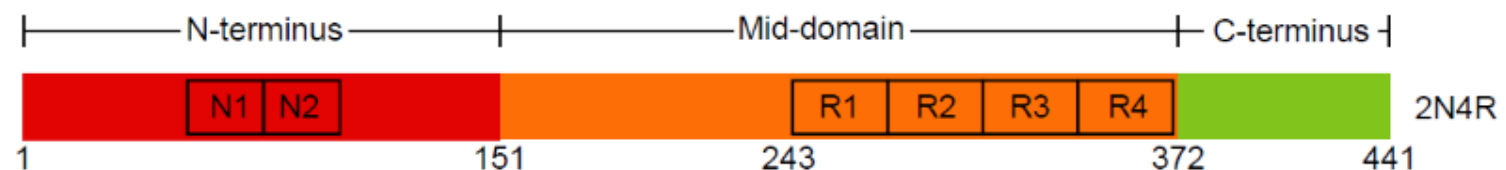

LY3303560

- (Zagotenemab)

7-9 and 312-342

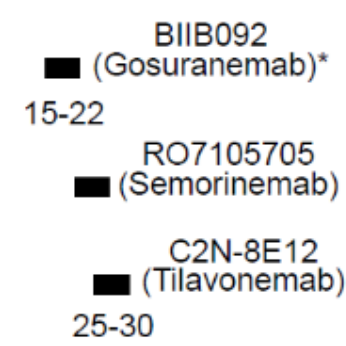

- BIIB076

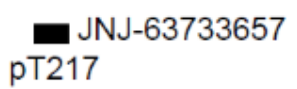

UCB0107 $235-246$

Figure 1: Tau antibodies currently in phase 1 or phase 2 trials in Alzheimer's disease.

Where known, the identity of the targeted epitope is included. *, discontinued Gosuranemab trial ${ }^{9}$. 
Author contributions: Dr Jabbari and Professor Duff contributed equally to the writing of this article.

Competing interests: Dr Jabbari was a study site sub-investigator in the Gosuranemab trial.

Professor Duff is a director and scientific board member of Ceracuity LLC.

\section{References}

(1) Golbe, L.I. \& Ohman-Strickland, P.A. A clinical rating scale for progressive supranuclear palsy. Brain 130, 1552-1565 (2007).

(2) Dam, T., et al. Safety and efficacy of the monoclonal anti-tau antibody Gosuranemab in progressive supranuclear palsy: the PASSPORT trial. Nat Medicine X, X-X (2021).

(3) Hoglinger, G.U., et al. Safety and efficacy of tilavonemab in progressive supranuclear palsy: a phase 2, randomised, placebo-controlled trial. Lancet Neurology 20, 182-192 (2021).

(4) Jadhav, S., et al. A walk through tau therapeutic strategies. Acta Neuropathol Commun. 7, 22 (2019).

(5) Sopko, R., et al. Characterization of tau binding by gosuranemab. Neurobiol Dis. 146, 105120 (2020).

(6) Yanamandra, K., et al. Anti-tau antibody reduces insoluble tau and decreases brain atrophy. Ann Clin Transl Neurol. 2, 278-288 (2015).

(7) Kim, B., et al. Tau immunotherapy is associated with glial responses in FTLD-tau. Acta Neuropathol. doi:10.1007/s00401-021-02318-y. Online ahead of print (2021).

(8) Jabbari, E., et al. Variation at the TRIM11 locus modifies Progressive Supranuclear Palsy phenotype. Ann Neurol. 84, 485-496 (2018).

(9) Biogen Shelves Gosuranemab After Negative Alzheimer's Trial.

https://www.alzforum.org/news/research-news/biogen-shelves-gosuranemab-after-negativealzheimers-trial (2021).

(10) Jabbari, E., et al. Genetic determinants of survival in progressive supranuclear palsy: a genomewide association study. Lancet Neurology 20, 107-116 (2021).

(11) Evans LD, Strano A, Campbell A, et al. Whole genome CRISPR screens identify LRRK2-regulated endocytosis as a major mechanism for extracellular tau uptake by human neurons. Preprint at https://www.biorxiv.org/content/10.1101/2020.08.11.246363v1 (2020).

(12) Myeku, N., et al. Tau-driven 265 proteasome impairment and cognitive dysfunction can be prevented early in disease by activating CAMP-PKA signalling. Nat Medicine 22, 46-53 (2016). 